\title{
Analysis of Digital Twin Definition and Its Difference from Simulation Modelling in Practical Application
}

\section{I.I. Krasikov and A. N. Kulemin}

Ural Federal University named after the first President of Russia B. N. Yeltsin, Russia, 620002, Yekaterinburg, Mira street, 21.

\section{Abstract}

The digital twin is widely known as a tool for digitalization of a product, but there is no common definition concerning this term. This article discusses the definition and utilization of digital twin. Areas of use, it's implementation in the product lifecycle and most importantly it's benefits. The lack of a standardized concept of a digital twin leads to a misunderstanding between mathematical models and digital twin. Several definitions of digital twin were analyzed and compared with the definition of mathematical model and simulation modelling. The basic concept of areas of use for

Corresponding Author:

I.I. Krasikov

ivan.krasikov@urfu.ru

Received: 5 March 2020

Accepted: 18 March 2020

Published: 8 April 2020

Publishing services provided by Knowledge E

(c) I.I. Krasikov and A. N.

Kulemin. This article is

distributed under the terms of

the Creative Commons

Attribution License, which

permits unrestricted use and

redistribution provided that the

original author and source are

credited.

Selection and Peer-review under the responsibility of the SEC 2019 Conference Committee. digital twin is introduced. The differences and similarities between the two definitions were found. The article aims first of all to help the management of digital twins in practical application.

Keywords: Digital twin, Mathematical modelling, Mathematical model, Lifecycle of a product, Simulation modelling, Practical use of digital twin, Difference between the digital twin and mathematical model, Simulation.

\section{Introduction}

Since its presence among the most promising technology trends in Gartner's recent report, the Digital Twin concept has become more popular in both academia and industry, apparently with many different attributes to include or exclude in its definition [1].

With the successful implementation of a digital twin in a lot of technical projects all around the world, the interest in this technology is constantly growing. But while there is no international standard for digital twin (ISOICD 23247-1 is only under development now) and every specialist in areas where digital twins could be used understand the definition in their own way, there would be troubles with the right implementation and connecting them in the future if we wouldn't make them standardized.

Nowadays a lot of companies try to make products which include a digital twin in it. Unfortunately, without the right understanding of the term, instead of digital twin 
companies develop mathematical models or produce simulation modelling of a product. This could lead the company to not fulfil the needs of the customer. And to the customer who doesn't understand how to do the verification and validation of a digital twin.

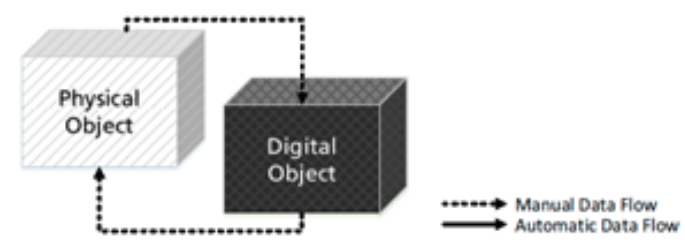

Simulation Modelling

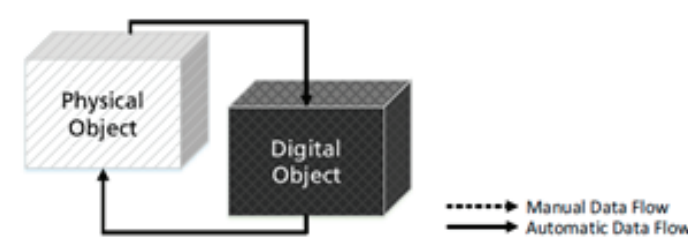

Digital Twin

Figure 1: Difference between Simulation Modelling and Digital Twin

\section{Purpose of the Paper}

The purpose of the paper is to make one basic definition of a digital twin and to divide this term from simulation modelling

This definition could be used by creators of a digital twin to be sure that their product will meet the needs of a customer. Also, for customers of a digital twin, this definition would be a tool to verification and validation of a product they own.

\section{Definitions}

Since the emergence of the idea of Industry 4.0 industry started to use digital twin term for marketing the numerous definitions have arisen, as some of them show:

The digital twin is a digital representation of things from the real world. Realistic digital representations of physical things. [2] A digitalized (3D) reproduction of a product to be created.

the vision of the digital twin itself refers to a comprehensive physical and functional description of a component, product or system, which includes all information which could be useful in all - the current and subsequent - lifecycle phases". [3]

There is a lot of definition of a digital twin which vary in many parameters such as degree of detail, technical focus or their scope. Basically, the digital twin is an asset of virtual models which are fully integrated with a real product.

Mathematical Model: A mathematical model is a description of a system using mathematical concepts and language. The process of developing a mathematical model is 


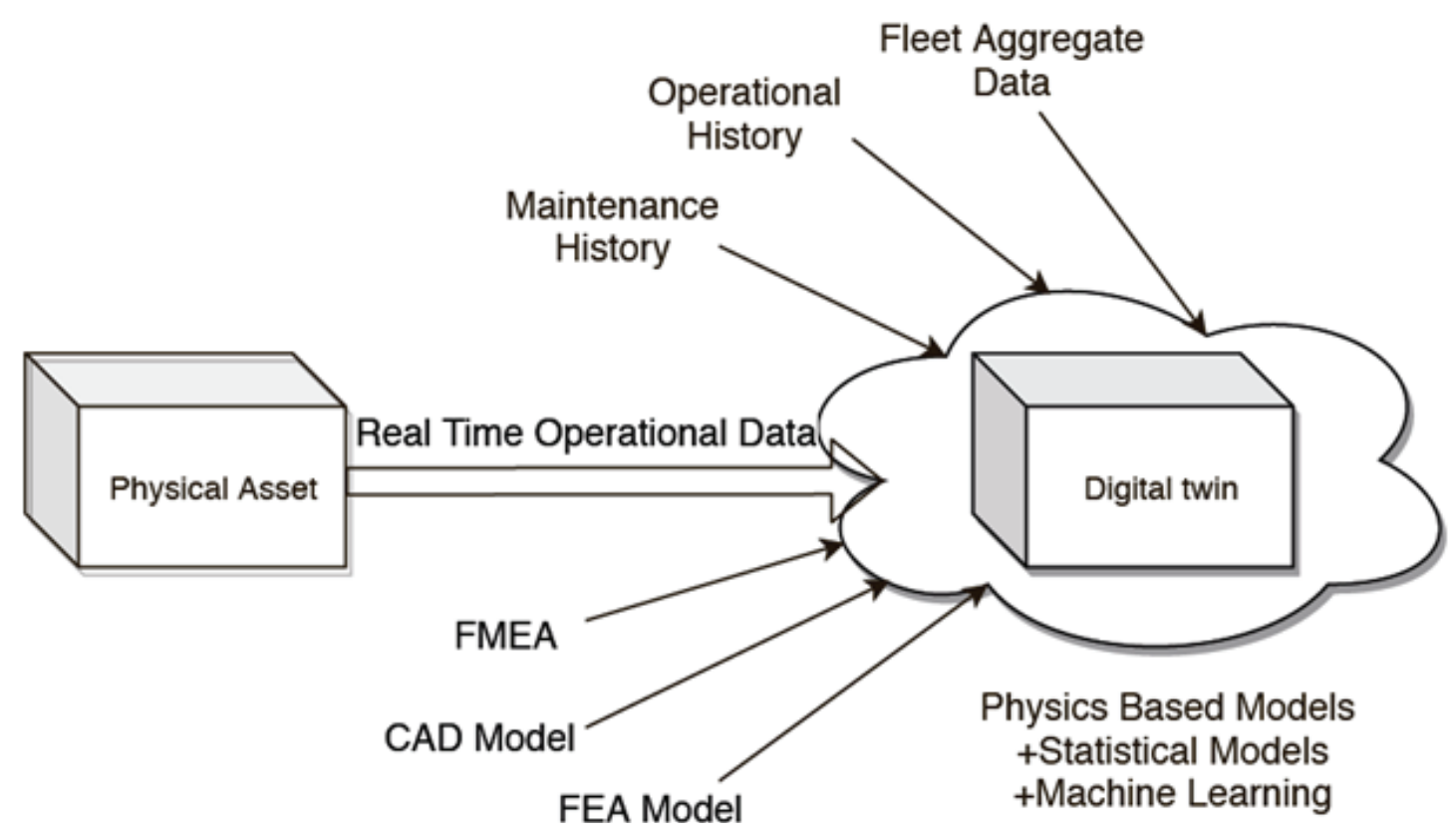

Figure 2: The Digital Twin - Physical Asset interaction

termed mathematical modelling. A model may help to explain a system and to study the effects of different components, and to make predictions about behaviour. [4]

Simulation: Simulation is the imitation of the operation of a real-world process or system over time. The act of simulating something first requires that a model be developed; this model represents the key characteristics or behaviours/functions of the selected physical or abstract system or process. The model represents the system itself, whereas the simulation represents the operation of the system over time. [4]

Simulation modeling is a type of mathematical modeling, the information form of images-models of which, manifested in coordinates (variables, relations) and parameters (coefficients, operators), has a physical meaning [5]

Simulation modelling: mathematical mode

\section{Similarities and Differences}

After we listed some digital twin definition and mentioned the definition of the mathematical model and simulation it would be logical to sort out the difference between them.

First, simulation modelling is usually used only for early stages of the lifecycle of a product like constructing and designing because of that modelling is used only by creators of a product. On the other hand, the digital twin is used not only for constructing the product, but it also made for customer to track the data of a product by using built-in 


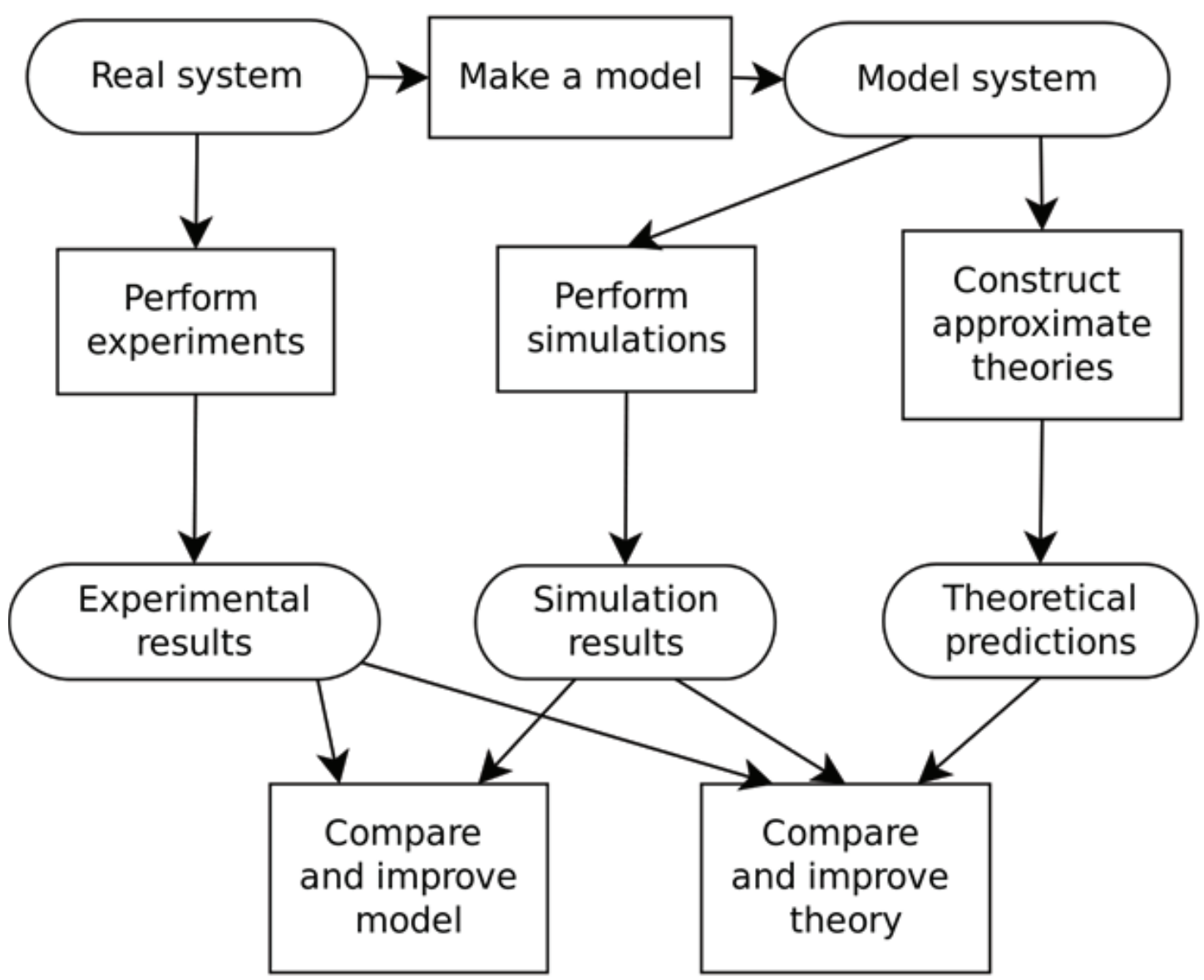

Figure 3: Simulation modelling

sensors and Internet of Things, it could predict using data from sensors and simulations the time when you would need to change the part or a product of a system.

Second, Simulations at best can help understand what may happen in the real world. Digital Twins not only help understand what may happen, but crucially what is happening (how the design is behaving in the real-world).

Third, Simulation modelling make the analysis of all processes inside the model the digital twin has the ability to recreate the interaction of an recreated object with others digital twins on a platform.

TABLE 1: Differences between Digital Twin and Simulation Modelling

\section{Digital Twin}

Used on every stage of a lifecycle

What is going on in the exact moment

Interactions between digital twins

\section{Simulation modelling}

Used on an early stage of the lifecycle (Designing and constructing)

What may happen

Only analyze the processes inside the model 


\section{Conclusion}

This paper is an analysis of a basic difference between the terms digital twin and simulation modelling and the difference in their application in practice. The main differences are that Digital Twin instead of simulation modelling is being used not only to create the product but to implement and use it. So, the digital twin of a product is a new type of simulation which give the customer a lot of new tools to operate and maintain their system.

Research on Digital Twins is only starting, there is a need for a lot of future research works on relevant industrial applications and a stable ISO standard of a Digital twin to investigate and demonstrate the wide range of applications and benefits where the Digital Twin could express their potential.

\section{References}

[1] B. Hicks, "Industry 4.0 and Digital Twins: Key lessons from NASA,"

[2] Bolton A, Enzer M, Schooling J et al. 'The Gemini Principles: Guiding values for the national digital twin and information management framework' (c) 2018 Centre for Digital Built Britain and Digital Framework Task Group

[3] VDI/VDE-GMA Fachausschuss 7.21.,

[4] Krishna, Addepalli V. N. and Balamurugan M. "Security Mechanisms in Cloud computing-based Big Data." Handbook of Research on the loT, Cloud Computing, and Wireless Network Optimization. IGI Global, 2019. 165-195.

[5] Bondarevsky A.S., Lebedev A.B. Simulation modelling: Definition and technical realization // Fundamental Research. - 2011. - № 12-3. - C. 535-541; 